\title{
Health technology assessment in Malaysia
}

\author{
Sadasivan Sivalal \\ Monash University Sunway Campus
}

\begin{abstract}
Objectives: Malaysia, as a rapidly developing country, has been facing tremendous pressures in its attempts to maximize scarce resources. Despite this problem, Malaysia has made great strides in developing its health services, and has successfully provided good access to the population to healthcare services, reduced the incidence of many communicable diseases, and improved life expectancies and other global indices of health care, some of which are comparable to that of developed countries.

Methods: The Health Technology Assessment (HTA) Unit was set up in Malaysia in August 1995 in the Ministry of Health Malaysia and has since grown tremendously in size and resources. To date, forty-three in-depth assessments have been carried out, and the recommendations of these assessments were subsequently implemented. In addition, approximately 140 rapid assessment reports were produced in response to requests from policy and decision makers. HTA has been able to provide input into formulation of national and Ministry of Health Malaysia policies, and provide a basis for clinical practice guidelines development, input into purchasing decisions, regulation of drugs, as well as advertisements related to health.
\end{abstract}

Results: A major challenge is sustainability of the program, to be able to have trained personnel competent to take on the demanding tasks of assessments and the sustained efforts that are required. In addition, there need to be constant efforts to create awareness of the utility of HTA so that its services are used and its full potential realized. The scope of services may also need to be expanded to include an early warning system.

Conclusions: Malaysia has successfully implemented a health technology program that has had major impact on policy formulation and decision making at various levels.

Challenges may be faced in sustaining and developing the program further.

Keywords: Health technology assessment, History, Malaysia, Health policy

Malaysia, like many other rapidly developing countries, is struggling with the dual objectives of achieving widespread and equitable coverage of health services while providing health care more efficiently under tight budget constraints. The challenges faced in this endeavor include rapidly escalating healthcare costs, the demographic shift to a more aged population, the availability of rapidly increasing expensive technological advances in the market, professional expectations, and increasing healthcare demand from the more informed patients. In the 1990s, Malaysia had already substantially increased the technological base of its healthcare

The author thanks all those who had provided him assistance in setting up and subsequently implementing the HTA program. He also thanks Dr. David Banta for mentoring him, and providing the support and encouragement, and in the process, becoming a dear friend. system mainly through the importation of medical equipment and pharmaceuticals. Concerns had been raised then about the need for consideration of benefit and cost-effectiveness (1). In addition, Malaysia embarked on technology transfer commencing with the turnkey concept of building several nuclear hospitals using expertise from the United Kingdom. However, while, on the whole, the process had gone quite well, some problems began to surface. This study will describe the attempts made to address these problems, especially in relation to how health technology assessment (HTA) came into being in Malaysia.

The material in this study is gathered from the author's knowledge of the situation, having been responsible for the acquisition of expensive medical equipment for hospitals at the national level and subsequently for the planning and setting up HTA in Malaysia. 


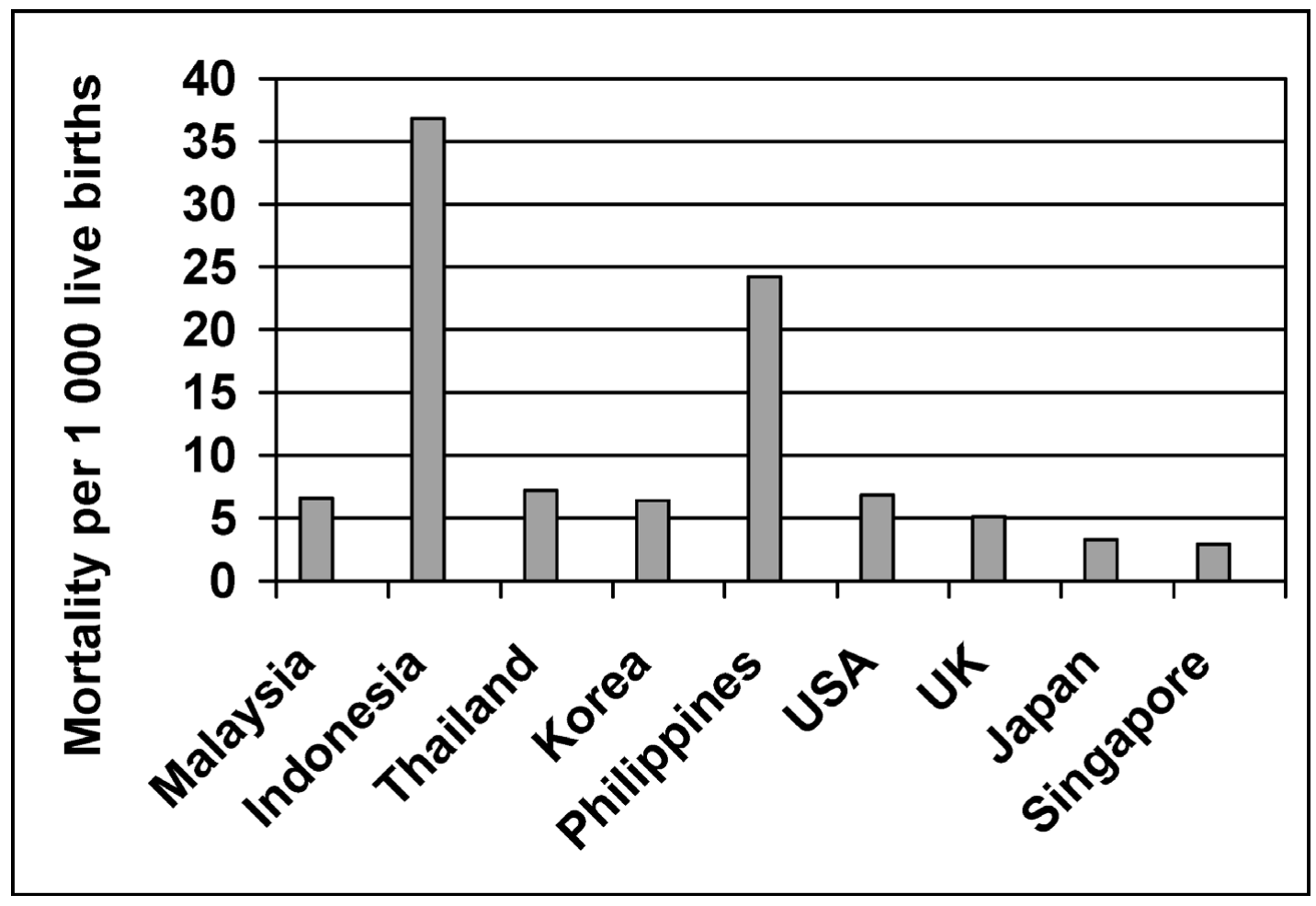

Figure 1. Infant mortality rates of selected countries.

Before describing the scenario leading to the establishment of HTA in Malaysia, some understanding of the healthcare system of Malaysia will be helpful.

\section{HEALTH CARE IN MALAYSIA}

Malaysia is situated in South East Asia, consisting of a peninsula continuous with the south of Thailand, and linked by a causeway to the island of Singapore at its southern tip. Apart from this, it also consists of the northern part of the island of Borneo, sharing it with Indonesia. It has a multi-ethnic population of approximately 27 million, consisting mainly of Malays, Chinese, and Indians. It obtained independence from the British in 1957, and has inherited many legacies from the British, including the administrative and legal systems.

Health care in Malaysia is mainly publicly funded with a network of healthcare facilities throughout the country, mostly under the Ministry of Health Malaysia. It is estimated that 78 percent of hospital beds are in public hospitals, whereas the rest are in private hospitals, located mainly in the large towns. Ambulatory health care is provided by general practitioner clinics, mostly in the urban areas, and community clinics in both urban and rural areas. However, it is estimated that approximately 60 percent of outpatients are seen by the private general practitioner clinics (3). There is no universal health insurance in Malaysia, but the public sector is heavily subsidized by the government, so that public health care is virtually free. The private health sector, on the other hand, functions on a fee-for-service basis. Members of the civil service are entitled to free public health care, whereas large employers have private health insurance schemes for their employees using mostly the private sector.

Malaysia has an effective healthcare system in that, with a relatively low level of expenditure, facilities are easily accessible, and the health statistics are comparable to countries with higher levels of economic development. It is estimated that 92 percent of the urban population live within $3 \mathrm{~km}$ of a health facility, whereas in rural areas, 69 percent in Peninsular Malaysia, 67 percent in Sabah, and 50 percent in Sarawak lived within $3 \mathrm{~km}$ of health facility (6). The life expectancy at birth in Malaysia is 71.8 years for males and 76.3 years for females. There has also been significant improvement in health care over the years - the crude death rate has decreased from 12.4 in 1957 to 4.5 per 1,000 live births, the maternal mortality ratio has dropped to 3 per 1,000 live-births, while the toddler mortality rate ( $1-4$ years) is currently 0.5 per 1,000 toddler population. The infant mortality rate has also shown a large decrease over the past 30 years from 75.5 in 1957 to 6.6 per 1,000 live births (4). This compares favorably with many countries in the region as well as some developed countries (2) as seen in Figure 1 below.

Much of the improvement in health care has been attributed to the emphasis on primary health care and health 
promotion and preventive activities (3). For example, immunization rates have been consistently high with rates of 98.9 percent for Bacillus Calmette-Guérin, 96.4 percent for third dose of Diphtheria/Pertussis/Tetanus, and 96.2 percent for third dose of polio. Poliomyelitis was eradicated in Malaysia in 2002. Public health activities at the district level include maternal and child health services, control of communicable and noncommunicable diseases, environmental sanitation services including provision of clean drinking water, sewage and waste disposal, food quality control, and occupational health and safety.

With respect to disease patterns, communicable diseases are generally at a low level with the exception of dengue, whereas some diseases such as tuberculosis, malaria, and hand, foot, and mouth disease have shown resurgences. Considering hospitalization, apart from pregnancy and childbirth related reasons, the major causes include accidents, diseases of the respiratory system, and diseases of the circulatory system. Similarly, the major causes of deaths in hospitals are septicemia, heart disease and diseases of pulmonary circulation, malignant neoplasms, cerebrovascular disease, pneumonia, and accidents (3).

As mentioned previously, the level of expenditure for health has been relatively low, with approximately 6.3 percent of the total national budget being allocated to the Ministry of Health Malaysia in 2006 (7). For example, operating expenditure in 2006 was RM7.4 billion compared with RM4.5 billion in 2001, whereas the development budget has remained prudently low, increasing only slightly from RM1.2 billion in 2001 to RM1.3 billion in 2006 (7).

Sophisticated medical equipment was also introduced into hospitals in a planned and systematic manner with phased implementation. The acquisition of this equipment was centralized at the Ministry of Health Malaysia to not only allow for economies of scale, but also to ensure that the level of equipping was appropriate to the level of services being provided and in keeping with the expertise available. For example, if cardiology services were being planned to be set up, facilities would be set up as and when manpower became available, because the placement of consultants was also carried out centrally. Standard equipment lists were also created to designate the minimum types and numbers of equipment for specific types of disciplines and services. Considering renal dialysis as an example, hemodialysis machines were first introduced in Ministry of Health hospitals by a donation of nine units from a nongovernmental organization in 1984. The numbers of units steadily increased to nineteen in 1991 and twenty-six in 1995. However, with the increasing numbers of nephrologists available as well as the increased demands for such services, hemodialysis units were soon available in all large- to medium-sized hospitals, and even in some of the smaller hospitals. Apart from this, these services were also available in the private sector for those who could afford to pay for them. Nonetheless, demands far outstripped service and it was soon realized that other strategies had to be adopted. Hemodialysis services were then made available in centers outside hospitals through the auspices of the National Kidney Foundation, a nongovernmental organization, with the assistance of the Ministry of Health Malaysia.

One effective strategy that was adopted was the regionalization of expensive and technology intensive services such as cardiology, radiotherapy, nuclear imaging, joint replacement, and the like. A referral system in the Ministry of Health Malaysia hospitals ensures that every patient obtains the level of health care appropriate to type and severity of the disease or condition for which he seeks treatment. Heart and liver transplants have also been carried out in Malaysia.

Decisions in relation to introduction of technology in health care have largely been sound. Traditionally, they depended on plans and policies with input from medical consultants from various specialties. However, the rapid advances in technology made it difficult for these consultants to keep abreast of the latest modalities. In addition, overzealousness in setting up centers of excellence or attempts to be leading centers in specific areas of expertise did give rise to some hasty decisions. Apart from these problems, the Ministry of Health Malaysia had no control over the acquisition of technology in the private sector. For example, the acquisition of an extracorporeal shockwave lithotripter attachment for the treatment of gall bladder stones proved to be ineffective, although the lithotripter worked for kidney stones. The electron beam computed tomography scan has not been shown to improve outcomes in the diagnosis of people with potential cardiac problems. Similarly, the transmyocardial laser for the treatment of coronary artery thrombosis has not been proven to be clinically effective. In addition, practices that have not been shown to be of benefit such as routine medical examination, routine chest X-rays, routine preoperative investigations, and antenatal electronic fetal monitoring continued to be carried out.

Thus, it became increasingly evident that there was a need for a more effective mechanism for the selection and introduction of technologies into the healthcare system. In addition, there were a few factors that provided added impetus. First among these was the economic boom of the early 1990s, which translated to having much increased resources to acquire sophisticated technologies as soon as they were available in the market. This spurred healthcare care providers to make increasing demands "get the latest" and "get the best," purportedly to ensure that their patients got the best possible care. The economic boom also resulted in the mushrooming of private hospitals, especially in the larger cities and towns. They were using technology as a means of competition with each other, equating sophisticated equipment with high quality care. Finally, with increasing awareness among the public, increasing availability of the Internet, and also increased Internet literacy, the public began to make demands for technology that was available in the private sector to be made available in the public hospitals as well. 
The Ministry of Health Malaysia was thus spurred to plan a, HTA unit in 1995. Dr. S. Sivalal, who had previously worked in the Quality Assurance Unit, and subsequently had been responsible for the acquisition of sophisticated medical equipment in the Ministry of Health Malaysia, was designated this task of setting up HTA.

\section{SETTING UP OF HTA IN MALAYSIA}

Sivalal thus headed a one-person HTA unit beginning in August 1995. However, the unit subsequently grew, until to date, it has a considerable amount of manpower and other resources. As part of his initial training in HTA, Sivalal spent a week in the then Canadian Coordinating Centre for HTA (CCOHTA, now known as CADTH) and also visited the Quebec HTA agency, to learn the various aspects of HTA. He then spent a 6-week attachment in ECRI, Philadelphia in the United States to get "hands-on" training. During this period, he also visited the Office of Health Technology Assessment (the Office of Technology Assessment had just closed then) and the Food and Drug Administration in Washington to appreciate the range and scope of HTA, the various organizational models as well as the applications of HTA.

The Ministry of Health Malaysia then obtained the services of a consultant, Dr. H.D. Banta, to assist it in the task of developing HTA in the country. Banta visited both public and private hospitals as well as other health facilities, and had numerous discussions with Ministry of Health Malaysia personnel before recommending the setting up of a national program for HTA encompassing both public and private sectors. However, this could not be implemented at that time because existing laws lacked a mechanism for the government to exercise any control over the introduction of technology into the private sector. In addition, Malaysia did not regulate medical devices, although pharmaceuticals were well regulated. Subsequently, however, a new law, the Private Health Care and Facilities Act, was enacted in 1998, which empowered the Ministry of Health Malaysia, among other things, to control the introduction of selected technology in the private sector.

The scope of HTA was to include drugs, devices, medical, and procedures, and organizational and support systems through which health care is provided including such things as the electronic medical record and day care surgery. It was also to encompass both new as well as existing technology.

\section{ORGANIZATION OF HTA IN MALAYSIA}

The success of any HTA program is measured by the extent of input into policy formulation and decision making. To ensure this input, it was imperative that the HTA program be "visible," and thus it was decided to set up a HTA Council chaired by the Director General of Health (5). There was also a need to involve all those involved in the provision of health care in the country, and so it was decided to have as its members top policy makers and decision makers from the public, academic, and private sectors. Subsequently, when clinical practice guidelines (CPG) came under the purview of the HTA unit, the role of the Council was expanded to cover CPG as well. The Council was then renamed HTA \& CPG Council.

\section{Role of HTA Council}

The Council was meant to oversee the whole HTA program. Hence, the terms of reference of the Council were to identify priority issues for HTA, to review the HTA program, approve health technology assessments carried out and formulate policies related to technology, and to review the dissemination and implementation activities of HTA. However, subsequently, a decision was made to separate administrative and technical components of HTA, so that the Council wished to confine its role only to technical matters.

\section{Technical Advisory Committee}

To assist the Council, a Technical Advisory Committee was set up. This Committee was tasked with reviewing all health technology assessments and providing technical input to the Council. It was thought to include as its members those with expertise in medicine, public health, research design, quality assurance, and technology assessment.

\section{Expert Committees}

The actual assessments are carried out by expert committees appointed especially for the technology under consideration. An expert committee would essentially consist of all those involved in the use of the technology. In some situations, in-house assessments were also carried out where the assessments were carried out by personnel in the HTA Unit, either working with relevant clinicians or submitting their work for review by relevant clinical consultants.

\section{Role of HTA Unit}

Because the HTA unit is involved at all levels - the expert committee, Technical Advisory Committee, HTA Councilcontinuity is ensured. It also serves as a quality check because personnel from the Unit can assist and guide the expert committee, provide technical input to the Technical Advisory Committee, as well as provide clarification at the HTA Council where needed.

\section{HTA WORK PROCESS}

The following are the stages in the work process for HTA: (i) identification of HTA topics, (ii) specification of assessment problem, (iii) determination of scope/scale and mode of assessment, (iv) retrieval of evidence, (v), collection of primary data, (vi) analysis of evidence, (vii) synthesis of evidence, (viii) formulation of findings and recommendations, 
(ix) dissemination and implementation, and (x) monitoring and feedback.

\section{DEVELOPMENT OF HTA}

From the time HTA unit was set up in 1995, forty-three in-depth assessments have been carried out and the recommendations of these assessments subsequently implemented. Apart from this, approximately 140 rapid assessments have been carried out in response to request from various quarters.

The impact of these assessments has been in various ways, like the formulation of national and Ministry of Health Malaysia (MOH) policies, providing a basis for clinical practice guidelines development, input into purchasing decisions, regulation of drugs, as well as advertisements related to health. Some examples are as follows.

National policy - The national immunization program was revised, where new vaccines for measles/mumps/rubella and Haemophilus influenzae type b were introduced, and timings of the existing immunization schedule were changed. The use of the drug Misoprostol was found to be unsafe for use in termination of pregnancy and induction of labor and was not recommended.

$\mathrm{MOH}$ policy on drugs-For the medical management of benign prostatic hyperplasia, drug treatment was only recommended if the anticipated life expected of the patients was less than 10 years, because it would not be cost-effective otherwise. An ineffective drug therapy called autologous target cytokines as treatment for all forms of cancer was prevented from entering into the country, because the HTA highlighted the lack of evidence of efficacy.

- $M O H$ policy on services-An HTA recommended the introduction of a home care nursing service, which has been commenced in some hospitals; the scope of effectiveness of home visiting was reviewed and subsequently new strategies formulated. Minimal access surgery was introduced based on HTA on the basis of safety, effectiveness, and cost-effectiveness

- $\mathrm{MOH}$ policy on screening-Screening for congenital hypothyroidism has been implemented for almost all newborn babies in $\mathrm{MOH}$ facilities. It was decided not to carry out routine antenatal screening for congenital abnormalities, but to provide this service only on request. An organized screening program for diabetic retinopathy in diabetics has also been introduced. Similarly, routine antenatal ultrasound screening is not recommended, being confined to only high risk pregnancies. A program for screening for hearing loss in high risk infants has also been recommended. Routine screening for obesity in children is also not recommended, because HTA highlighted the lack of evidence of effective intervention, so that emphasis should be on prevention of obesity.

- Equipment purchasing decisions-purchasing decisions for medical equipment have also been based on HTA. For example, the gamma knife was not purchased on the basis of costeffectiveness, and instead, the existing linear accelerator was modified to be able to carry out stereotactic radiosurgery for small brain tumors. Dry chemistry analyzers were found to be cost-effective only for remote health centers and the flying doctor service in East Malaysia, and not for near patient testing or for wellness screening. The use of dry laser for processing digital radiological images was recommended to replace the older equipment of the wet method. An HTA on low temperature sterilization highlighted the safety concerns in some of the older methods of sterilization. Unproven technology such as electromagnetic prostatectomy was not recommended. A policy to prevent the introduction of new, prefabricated myofunctional appliances in dentistry was recommended. A policy was also formulated to discontinue the ineffective equipment of Venturi medical gas system, based on the HTA, which found a lack of evidence of efficacy

- MOH policy on procedures-Similarly, a policy was made to implement new proven effective procedures such as the use of polymerase chain reaction (PCR), spinal cord stimulation for relief of intractable pain, as well as minimal access surgery.

- MOH policy on practices-An HTA recommended that in the management of moderately elevated blood pressure, the initial treatment should be started with nonpharmacological methods before initiating drug therapy. There was a lack of evidence found on physiotherapy modalities used such as ultrasound, wax, and so on, on the issue of heat treatment of degenerative skeletal changes, sufficient evidence being obtained only for capsicum. Similarly, desferroxamine was found to be effective in reducing the iron overload due to long-term transfusion in Thalassemia management. There was insufficient evidence on the superiority of second-generation modalities for use in endometrial ablation. A policy was made to continue the effective practice of administration of vitamin $\mathrm{K}$ in newborns. On the other hand, a policy for not carrying out routine skull X-ray in minor head trauma has been implemented through a circular to $\mathrm{MOH}$ hospitals. There was also a policy recommending the elimination of routine chest X-ray in routine medical examination, and later of pre-employment routine medical examination.

Apart from these examples, HTA has been used as a basis for CPG development, for example, guidelines on the rational use of preoperative investigations, management of Thalassemia, rational antibiotic utilization in common pediatric conditions, and childhood immunization were all drawn up based on the assessment on these topics. A guideline as well as a clinical pathway on the management of Neonatal Jaundice were also drawn up based on the HTA of the condition.

\section{CPGs under the Purview of the HTA Unit}

CPGs were previously developed by various agencies, that is, Academy of Medicine, Malaysia, as well as professional bodies and societies. Most of the CPG developed were not systematic, and were often consensus based. In 2001, CPG were brought under the purview of the HTA Unit. Standardization of the development of CPG was instituted with a formal mechanism for the approval of topics for CPG, as well as for the approval of completed CPG before they were published. All draft CPG were placed on the Internet to allow 
wider opportunities for input. In addition, personnel from the relevant disciplines were invited to provide comments or give input where necessary, before the CPG were finalized.

\section{Web Page for HTA Unit}

A Web page for health technology assessment has been created to allow HTA services and products to be accessed through the Internet, as well as to improve the dissemination of information. The work process, summaries and full reports of all assessment carried out, as well as a list of technology reviews and ongoing assessments, are displayed.

\section{Training in HTA}

The HTA unit also strived to increase awareness by providing training on HTA. Training courses were held annually from 1996 for various healthcare providers such as specialists in hospitals, as well as personnel from Ministry of Health Malaysia (8). These were also open to personnel from other countries, and to-date, personnel from developing countries such as India, Iran, Indonesia, Pakistan, and Philippines have attended these courses. In addition, HTA seminars are held annually for allied health personnel, as well as nurses and medical assistants. Apart from this, seminars and continuing medical education sessions were conducted at state and hospital levels. Training on HTA was also provided to postgraduate students in the universities such as University of Malaya and University Kebangsaan Malaysia. Efforts were also made to extend the awareness to undergraduate medical students, for example, in the International Medical University. Consultancy services to other countries such as Indonesia, Iran, and Pakistan, which including training on HTA to personnel in those countries, were also provided

\section{World Health Organization Collaboration Center}

The HTA unit was designated as a World Health Organization (WHO) Collaborating Center for Evidence-Based Practice in Health Care for the Western Pacific Region from July 2004, and Sivalal was appointed Director of this center.

\section{International Conferences}

The first Asian Regional Health Technology Assessment Conference was held in collaboration with the International Society of Technology Assessment in Health Care (ISTAHC), World Health Organization, and the Malaysia Society of Quality Assurance in 2000. This conference provided a forum to exchange experiences on HTA in the region. Approximately 350 participants from twenty countries attended this conference. The second Asian Regional Health Technology Assessment Conference was held jointly with the World Health Organization and the Malaysian Society of Health Technology Assessment in 2003 with approximately 300 participants from twelve countries. In addition, an Asian Regional Evidence Based Nursing Conference was held jointly with Malaysian Society of Health Technology Assessment in 2003 with approximately 400 attendees.

\section{Asian Regional HTA Network}

The HTA Unit was also involved in the setting up of an Asian Regional HTA Network in 1996. This network aimed to share experiences, pool expertise, and generally foster the development of HTA in the region.

\section{Paper Presentations at Conferences}

Personnel from the HTA Unit presented numerous papers at conferences both locally and abroad. Papers have been presented at almost every ISTAHC and HTAi conference since 1997.

\section{IMPACT OF FUTURE DEVELOPMENTS IN HEALTH CARE ON HTA}

The Ministry of Health Malaysia is exploring alternative financing mechanisms for the provision of health care. It is envisaged that the HTA Unit will have a role in play in this scheme of things, especially in reference to decisions on benefit packages and reimbursements.

Regulation of medical devices is also being planned to be implemented in the near future. This is expected to cover manufacture, sale, use, and maintenance of medical devices in the country. HTA is also expected to play an important role in this area, especially in relation to registration of new devices.

Another area of possible contribution is the Quality Assurance program where standards have been created for accreditation as well as for monitoring quality of care. HTA may be able to provide a research basis for these standards and so make them more robust and acceptable.

Related to this is the implementation of various provisions of the Private Health Care and Facilities, which include the location of facilities as well as the use of various technologies. Input from the HTA Unit may be needed on the types of devices to be monitored, as well as possible numbers and placements of these devices.

\section{DISCUSSION}

Whereas the HTA program has enjoyed considerable success, a major challenge is the issue of sustainability. Some key personnel involved in the program from the outset are no longer in the unit, and there is thus a shortage of well-trained, experienced personnel. Although newer staff is available, it may take considerable time and effort to provide them sufficient training to function effectively. This staff turnover continues to be a challenge, and major efforts and clear strategies are needed to overcome this to effectively sustain the program. 
Another related issue is the continued inadequate awareness of policy makers, decision makers, healthcare personnel, and the general public on HTA. Despite all efforts at increasing awareness so far, there is room for increased utilization of HTA services. Unless these are not only continued and sustained, but possibly upgraded, it may be difficult to sustain the utilization of the services being offered by the HTA Unit.

Finally, there is a need to increase the scope of the HTA Unit to encompass more areas such as, for example, instituting early warning system or horizon scanning to provide awareness to healthcare providers and the public on new technologies.

\section{CONCLUSION}

Malaysia has successfully implemented a health technology program, which has been able to provide considerable input into policy formulation and decision making at national level as well at the levels of the Ministry of Health Malaysia and healthcare facilities. It may face challenges in sustaining the program and further moving it forward. It may also be worthwhile to consider an external review of the HTA program in an effort to further improve it to benefit policy makers, decision makers, healthcare providers, and the public. The program has been able to involve the medical profession in its activities, and is not at all controversial with the profession.

\section{CONTACT INFORMATION}

Sadasivan Sivalal, MBBS, PGDHHSA (sivalal@hotmail. com), Senior Lecturer, School of Medicine \& Health Sciences, Monash University Sunway Campus, Jalan Lagoon Selatan, 56150 Bandar Sunway, Selangor, Malaysia

\section{REFERENCES}

1. Bakar A. Working for health. Kuala Lumpur: Ministry of Health Malaysia; 1995.

2. Ministry of Health Malaysia. My health: Health status of Malaysia. Kuala Lumpur: Ministry of Health Malaysia; 2005.

3. Ministry of Health Malaysia. Annual Report Ministry of Health Malaysia 2006. Kuala Lumpur: Ministry of Health Malaysia; 2007.

4. Ministry of Health Malaysia. Indicators for monitoring and evaluation of strategy for health for all. Kuala Lumpur: Ministry of Health Malaysia; 2006.

5. Ministry of Health Malaysia. Malaysia's health 2000. Kuala Lumpur: Ministry of Health Malaysia; 2000:146-158.

6. Ministry of Health Malaysia. National Health Morbidity Survey II. Kuala Lumpur: Ministry of Health Malaysia; 1996.

7. Ministry of Health Malaysia. www.moh.gov.my (accessed September 30, 2008).

8. Sivalal S, Banta D, T'Hoen E, Jaudin R. Training in health technology assessment. Int J Technol Assess Health Care. 1998; 14:809-817 